\title{
On the accuracy of intracardiac flow velocimetry methods
}

\author{
Arash Kheradvar $^{1}$
}

Received: 6 January 2017 / Accepted: 9 January 2017/Published online: 15 February 2017

(C) Japanese Society of Echocardiography 2017

Keywords Vortex imaging $\cdot$ Vector flow mapping $\cdot$ EchoPIV · Velocimetry · Fluid dynamics · Flow visualization

"Begin challenging your own assumptions. Your assumptions are your windows on the world. Scrub them off every once in a while, or the light will not come in."-Alan Alda [1].

The topic of the flow pattern inside the heart and vortex imaging has been a main stream of research in echocardiography during the past decade. Progress has been made to incorporate quantitative fluid dynamics into echocardiography using particle tracking algorithms $[2,3,39]$ that are based mostly on the well-known optical imaging techniques of particle image velocimetry (PIV) [4-6] or color Doppler imaging [7-10]. Recent advances in understanding left ventricular (LV) fluid dynamics based on experimental methods [11-14] and numerical simulations [15-17] have shed light on many aspects of ventricular flow, such as the development of intraventricular vortices. These vortices are shown to significantly influence transmitral momentum transfer and help redirect the flow from the left atrium toward the left ventricular outflow tract (LVOT) [18, 19]. Alternatively, formation of unnatural vortices can be a sign of adverse blood flow, which may indicate progressive LV dysfunction [18-21]. The knowledge gained about LV fluid dynamics, and in particular the associated vortical flow motion, has introduced novel

Arash Kheradvar

arashkh@uci.edu

1 The Edwards Lifesciences Center for Advanced Cardiovascular Technology, University of California Irvine, 2410 Engineering Hall, Irvine, CA 92697-2730, USA clinical indicators for LV function based on vortex dynamics [18, 19, 21-25].

PIV is an optical method for flow visualization used to obtain instantaneous velocity measurements and related properties in the fluids. In this technique, the fluid is seeded with tracer particles, which are assumed to faithfully follow the dynamics of flow. The motion of these seeding particles is used to compute the flow velocity. In its current form, 2D ultrasound-based PIV or 2D echocardiographic PIV (EchoPIV) was introduced by Kim et al. [2], through capturing digital B-mode images of contrast agent particles, and further used for vortex imaging by Kheradvar et al. [21]. This technique computes the velocities of the ultrasoundimaged particles based on the PIV technique, with the $\Delta t$ being equal to scanning time. The number of beams and the samples along each beam define the number of pixels for each image after scan conversion. Particles used as the flow tracers are microbubbles filled with octafluoropropane encapsulated in either a lipid (DEFINITY ${ }^{\circledR}$, Lantheus Medical Imaging, Inc.) or protein $\left(\right.$ Optison $^{\mathrm{TM}}$, GE Healthcare) outer shell $[3,26]$, which are both FDA-approved for clinical use. This technique allows the velocity directions and streamlines, principal blood flow patterns, recirculation regions, and vortices to be drawn with reasonable confidence in a reproducible scheme [18, 21, 22, 27-32].

Alternatively, vector flow mapping (VFM) measures blood flow velocity by considering color Doppler imaging and ventricular wall velocity [7-10]. This method works based on combining measured axial velocities with estimated radial velocities according to the physical principles [33]. VFM ignores the three-dimensional component of the flow by assuming the flow is two-dimensional, solves the 2D continuity equation, and use ventricular wall velocity acquired by tissue tracking to improve the results [34]. 
In reality, any physical flow is three-dimensional. However, some flow regimens can be considered 2D if the out-of-plane velocity component does not (or at least minimally) exist. A good example for such a flow regime is laminar flow in an axisymmetric tube. In laminar flow, there is no lateral mixing, and the nearby layers pass each other in a totally parallel scheme. Laminar flow requires no cross-currents perpendicular to the flow direction or eddies/ swirls in the fluid [35]. Non-uniform geometries, such as in the heart chambers, increase flow three-dimensionally. Furthermore, time-dependency and the rotational nature of the flow minimize the application and accuracy of the methods developed for potential flow. Principles of fluid dynamics should be properly considered and applied for each particular flow regimen to avoid fundamental oversights in solving cardiovascular problems [36].

In prospect, intracardiac flow velocimetry is an emerging field in cardiac imaging. It should be considered that intracardiac flow is principally three-dimensional, timedependent, and non-laminar. Modern echocardiography systems use ultrasound probes that can capture three-dimensional brightness fields associated with the blood flow. Generally, the ultrasound-based velocimetry methods are all bounded by the limitations and constraints of echocardiographic acquisitions, such as inter and intra-operator variabilities and acoustic shadowing. Furthermore, limited frame rate of echocardiographic acquisitions-particularly in 3D-is currently a major obstacle for accurate assessment of high-velocity values and advancement of 3D ultrasound-based velocimetry modalities for intracardiac flow [21, 33, 37]. More recent efforts may overcome these limitations and pave the way for routine clinical applications $[33,37-39]$.

\section{Compliance with ethical standards}

Conflict of interest Prof. Kheradvar holds multiple pending patents on velocimetry methods.

\section{References}

1. Alan Alda, Thinkexist.com, Alan Alda quotes, Thinkexist. http:// thinkexist.com/quotation/begin_challenging_your_own_assump tions-your/221133.html Accessed 29 Dec 2016.

2. Kim HB, Hertzberg JR, Shandas R. Development and validation of echo PIV. Exp Fluids. 2004;36:455-62.

3. Zheng H, Liu L, Williams L, et al. Real time multicomponent echo particle image velocimetry technique for opaque flow imaging. Appl Phys Lett. 2006;88:261915-400000.

4. Adrian RJ. Particle-imaging techniques for experimental fluid mechanics. Annu Rev Fluid Mech. 1991;23:261-304.

5. Willert CE, Gharib M. Digital particle image velocimetry. Exp Fluids. 1991;10:181-93.

6. Falahatpisheh A, Kheradvar A. High-speed particle image velocimetry to assess cardiac fluid dynamics in vitro: from performance to validation. Eur J Mech B Fluids. 2012;35:2-8.
7. Ohtsuki S, Tanaka M. The flow velocity distribution from the Doppler information on a plane in three-dimensional flow. J Vis. 2006;9:69-82.

8. Garcia D, Alamo JCd, Tanne D, et al. Two-dimensional intraventricular flow mapping by digital processing conventional color-Doppler echocardiography images. IEEE Trans Med Imaging. 2010;29:1701-13.

9. Keiichi I, Takashi O, Tokuhisa U, et al. Intraventricular flow velocity vector visualization based on the continuity equation and measurements of vorticity and wall shear stress. Jpn J Appl Phys. 2013;52:07HF16.

10. Uejima T, Koike A, Sawada $\mathrm{H}$, et al. A new echocardiographic method for identifying vortex flow in the left ventricle: numerical validation. Ultrasound Med Biol. 2010;36:772-88.

11. Kheradvar A, Kasalko J, Johnson D, et al. An in vitro study of changing profile heights in mitral bioprostheses and their influence on flow. ASAIO J. 2006;52:34-8.

12. Domenichini F, Querzoli G, Cenedese A, et al. Combined experimental and numerical analysis of the flow structure into the left ventricle. J Biomech. 2007;40:1988-94.

13. Kheradvar A, Gharib M. On mitral valve dynamics and its connection to early diastolic flow. Ann Biomed Eng. 2009;37:1-13.

14. Kheradvar A, Milano M, Gharib M. Correlation between vortex ring formation and mitral annulus dynamics during ventricular rapid filling. ASAIO J. 2007;53:8-16.

15. Domenichini F, Pedrizzetti G, Baccani B. Three-dimensional filling flow into a model left ventricle. J Fluid Mech. 2005;539:179-98.

16. Pedrizzetti G, Domenichini F. Nature optimizes the swirling flow in the human left ventricle. Phys Rev Lett. 2005;95:108101.

17. Schenkel T, Malve M, Reik M, et al. MRI-based CFD analysis of flow in a human left ventricle: methodology and application to a healthy heart. Ann Biomed Eng. 2007;37:503-15.

18. Kheradvar A, Assadi R, Falahatpisheh A, et al. Assessment of transmitral vortex formation in patients with diastolic dysfunction. J Am Soc Echocardiogr. 2012;25:220-7.

19. Panupong J, Anna MC, Mohsen SA, et al. Impact of acute moderate elevation in left ventricular afterload on diastolic transmitral flow efficiency: analysis by vortex formation time. J Am Soc Echocardiogr. 2009;22:427-31.

20. Kheradvar A, Assadi R, Jutzy KR, et al. Transmitral vortex formation: a reliable indicator for pseudonormal diastolic dysfunction. J Am Coll Cardiol. 2008;51:A104.

21. Kheradvar A, Houle HH, Pedrizzetti G, et al. Echocardiographic particle image velocimetry: a novel technique for quantification of left ventricular blood vorticity pattern. J Am Soc Echocardiogr. 2010;23:86-94.

22. Hong G-R, Pedrizzetti G, Tonti G, et al. Characterization and quantification of vortex flow in the human left ventricle by contrast echocardiography using vector particle image velocimetry. JACC Cardiovasc Imaging. 2008;1:705-17.

23. Gharib M, Rambod E, Kheradvar A, et al. Optimal vortex formation as an index of cardiac health. Proc Natl Acad Sci. 2006;103:6305-8.

24. Poh KK, Lee LC, Shen L, et al. Left ventricular fluid dynamics in heart failure: echocardiographic measurement and utilities of vortex formation time. Eur J Echocardiogr. 2011;13(5):385-93.

25. Belohlavek M. Vortex formation time: an emerging echocardiographic index of left ventricular filling efficiency? Eur Heart $\mathrm{J}$ Cardiovas Imaging. 2012;13:367-9.

26. Liu LL, Zheng HR, Williams L, et al. Development of a customdesigned echo particle image velocimetry system for multicomponent hemodynamic measurements: system characterization and initial experimental results. Phys Med Biol. 2008;53:1397-412. 
27. Sengupta PP, Khandheria BK, Korinek J, et al. Left ventricular isovolumic flow sequence during sinus and paced rhythms: new insights from use of high-resolution Doppler and ultrasonic digital particle imaging velocimetry. J Am Coll Cardiol. 2007;49:899-908.

28. Eriksson J, Dyverfeldt P, Engvall J, et al. Quantification of presystolic blood flow organization and energetics in the human left ventricle. Am J Physiol Heart Circ Physiol. 2011;300:H2135-41.

29. Garcia D, del Alamo JC, Tanne D, et al. Two-dimensional intraventricular flow mapping by digital processing conventional color-Doppler echocardiography images. IEEE Trans Med Imaging. 2010;29:1701-13.

30. Luo J, Konofagou EE. Imaging of wall motion coupled with blood flow velocity in the heart and vessels in vivo: a feasibility study. Ultrasound Med Biol. 2011;37:980-95.

31. Pedrizzetti G, Domenichini F, Tonti G. On the left ventricular vortex reversal after mitral valve replacement. Ann Biomed Eng. 2010;38:769-73.

32. Zhang F, Lanning $\mathrm{C}$, Mazzaro $\mathrm{L}$, et al. In vitro and preliminary in vivo validation of echo particle image velocimetry in carotid vascular imaging. Ultrasound Med Biol. 2011;37:450-64.
33. Sengupta PP, Pedrizzetti G, Kilner PJ, et al. Emerging trends in CV flow visualization. JACC Cardiovas Imaging. 2012;5:305-16.

34. Asami R, Tanaka T, K-i Kawabata, et al. Accuracy and limitations of vector flow mapping: left ventricular phantom validation using stereo particle image velocimetry. J Echocardiogr. 2016. doi:10.1007/s12574-016-0321-5.

35. Geankoplis CJ. Transport processes and separation process principles. 4th ed. New Jersey: Prentice Hall Press; 2003.

36. Pedrizzetti G, Vlachos PP, Little WC, et al. Comments on defining the contribution of diastolic vortex ring to left ventricular filling. J Am Coll Cardiol. 2015;65:2573.

37. Falahatpisheh A, Kheradvar A. Abstract 14952: volumetric echocardiographic particle image velocimetry (V-Echo-PIV). Circulation. 2014;130:A14952.

38. Falahatpisheh A, Kheradvar A. A framework for synthetic validation of 3D echocardiographic particle image velocimetry. Meccanica. 2017;52:555-61.

39. Falahatpisheh A, Pedrizzetti G, Kheradvar A. Three-dimensional reconstruction of cardiac flows based on multi-planar velocity fields. Exp Fluids. 2014;55:1-15. 